\title{
Near misses: Nurses' experiences with medication errors, power distance and error recover
}

\author{
Luanne Linnard-Palmer, Tham Ngo \\ Dominican University of California, CA, United States
}

Received: July 29, 2015

DOI: $10.5430 / \mathrm{cns} . v 4 \mathrm{n} 1 \mathrm{p} 40$
Accepted: November 9, 2015

Online Published: December 2, 2015

\begin{abstract}
Background: Medication errors continue to be a major problem in health care settings in the United States. Research investigating factors contributing to medication errors continues in an attempt to reduce the incidence and save lives. Although electronic medical records and electronic prescribing technology have been reported to reduce the incidence, errors are continuing, some with significant impact on patient safety. A near miss is an incident that could potentially cause harm, was identified early and a mistake prevented. Little is known about near misses including the identification, intervention, prevention, and recovery. Nurses' perceptions, what led up to the incident, and how they went about correcting these incidents (recovery) provides insight to understanding what near misses are, where they take place, and how. Poor communication is directly linked to medication errors and near misses. In nursing, one communication variable that relates to errors and near misses concerns the concept of Power Distance and how it affects communication between nurses, colleagues and families. Power distance is based on a perception of inequality existing between individuals. This perception of power inequality may contribute to errors associated with communication such as near misses, actual errors, poor adherence to treatment and poor compliance with medications.

Objectives: The aim of this study was to investigate nurse's experiences of errors, recovery processes, the concept of Power Distance and poor communication that have led to near-miss incidents.

Methods: An on-line survey, developed for this mixed-methodological study, was completed by a total of 110 participants.

Results: Analysis of the descriptive data demonstrated three general themes of types of near misses experiences: wrong medication to wrong patients; error caught just before reaching patient; and recognition of error, correction made before implementation. Thematic analysis disclosed six themes related to actual examples of near misses: patient identified the near miss; medication was already administered by another nurse; a systems error occurred with the computerized dispending unit; the five rights were breeched; timing of administration was incorrect; and the wrong medication was dispensed directly from the pharmacy. Three themes emerged concerning miscommunication and near misses: between healthcare team members; between shifts; and pharmacy verifying and approving wrong med/wrong dose. Power distance was only minimally found to be of concern to the participants in relation to near misses.

Conclusions: Nurses' experiences with near misses, recovery and Power Distance illuminate the need to further investigate what recovery behaviors and prevention practices are taking place. Further knowledge in this area may improve communication, reduce errors and ultimately increase patient safety.
\end{abstract}

Key Words: Near miss, Medication error, Power Distance, Error recovery, Error prevention, Descriptive research design, Nursing care, Adverse results, Nursing practice

*Correspondence: Luanne Linnard-Palmer; Email: linnard@ dominican.edu; Address: Dominican University of California, 382 Irwin St, San rafael, CA 94901, United States. 


\section{BACKGROUND AND SIGNIFICANCE}

Medication errors continue to be a major problem in health care settings the United States. ${ }^{[1-3]}$ Research continues to investigate factors that contribute to medical errors. Many studies have investigated the issue of medication errors since the Institute of Medicine (2000) report "To Err Is Human" was published. ${ }^{[4]}$ There was a substantial increase in the number of patient safety publications and research awards following the release of the report. ${ }^{[5]}$ Near misses, on the other hand, are defined as an incident that could potentially cause harm to the patient, however the potential error was prevented. Little is known about near misses. With minimal success, many literature sources have proposed common definitions to what defines a near miss. However, there are numerous interpretations of near misses, which make it difficult to generalize. Many factors contribute to the development of a near miss including individual factors, system factors, and chaotic environments (see Table 1 for a list of the most commonly reported contributors to medication errors). Because of a lack of a single widely-accepted definition of near misses, research on the phenomenon is warranted. The purpose of this research was to investigate nurses' experiences with medication errors, recovery processes, Power Distance and poor communication that led to near miss incidents.

Table 1. Top reported elements that contribute to medications errors

Documentation/transcription errors
Communication errors
Computation errors
Workflow disruption
Distraction
Shift change
Patient transfers
Incomplete handoffs
Protocols not followed
Inadequate monitoring of patient
Abbreviation use
Verbal orders used
Labeling errors
Improper use of pumps

Nurses' perceptions on near misses, including what led up to the incident, and how they went about correcting these incidents provide great insight to understanding the occurrence. Nurses can be held responsible for causing errors, so it is important that they understand the consequences of these mistakes and how to prevent them. ${ }^{[6]}$ Since communication continues to be a significant variable contributing to errors and near misses, the concept of Power Distance, or perceived inequality, and how it effects communication between nurses, colleagues, parents and families has been identified to be of significance.

Power Distance refers to the extent people accept the fact that inequality exists in society. ${ }^{[7,8]}$ As a result communication barriers may develop among individuals of varying power, which in turn impacts the non-verbal aspects of communication. ${ }^{[9]}$ When high Power Distance exists, subordinates or persons deemed to have less power tend to show more respect, are polite, and agree in the presence of the person with higher power. In a health care setting the practitioners have higher Power Distance, since they have expertise that a patient does not possess. Likewise, the concept of Power Distance also results in inequities and disparities in healthcare.

Previous research demonstrated that for nurses a prior negative experience with an individual clinician appeared to be a critical factor that diminished the nurse's desire to interrupt an error involving the same clinician. ${ }^{[10]}$ Similarly within a multigenerational Asian family, the elders may be consulted and included in decision making, since their age and hierarchy within the family accords them the higher status. ${ }^{[11-13]}$ "Common factors that negatively influenced nurses' ability to care safely for patients were intimidation by nurses and physician colleagues and poor communication". ${ }^{[10]}$ The Joint Commission describes how sentinel events are directly linked to communication. ${ }^{[34]}$

Researchers are shifting from focusing on medical errors to near misses. Near misses occur far more frequently than actual errors. ${ }^{[14]}$ This implies that more data can be collected in less time. Causal paths of near misses and accidents are likely to be similar, therefore by eliminating the causes of near misses, one could prevent actual medication error accidents. In the case of near misses where patients are not harmed, nurses might be less ashamed or embaressed of what happened and have less fear of litigation, which might positively influence their willingness to report near misses. ${ }^{[15]}$ Without a proper definition of near misses, data collection on incidents continues to be difficult.

Near miss is a concept that is not well understood and studied mainly because there is no universal definition for it. Many authors and researchers have offered definitions based on personal interpretation. Various definitions for near misses include:

- An event or circumstance that has the potential to cause an incident or critical incident but that did not actually occur due to corrective action and/or timely intervention. ${ }^{[16]}$

- An act of commission or omission that could have 
harmed the patient but was prevented from completion through a planned or unplanned recovery. ${ }^{[17]}$

- Any event that could have had adverse consequences but did not and was indistinguishable from fully fledged adverse events in all but outcome. ${ }^{[18,19]}$

- Errors that had the capacity to cause injury failed to do so, either by chance or because they were intercepted. ${ }^{[20]}$

- An error of commission or omission that could have harmed the patient, serious harm did not occur as a result of chance, prevention, or mitigation. ${ }^{[21]}$

An example of a near miss given by ${ }^{[15]}$ was described by a nurse as: "the cardiology assistant physician had written a drug prescription without indication how often the drug should be administered. I showed the prescription to the internal medicine assistant physician, who completed it." This example demonstrated that the nurse recognized that the assistant physician did not indicate the frequency of the medication; therefore, the nurse corrected that error by clarifying it with the assistant physician. Another example of a near miss would be, "A nurse was passing out her scheduled medications and right before she was about to give a patient their pills, she realized she grabbed the wrong medication when going through the 'five rights'. She caught her mistake in time and no harm was done to the patient" (Personal Communication, April 2, 2014, Ngo). The aim of this study was to investigate nurse's perceptions and experiences of errors, recovery processes, the concept of Power Distance, and poor communication that have led to near-miss incidents.

\subsection{Research questions}

- How do nurses describe their experiences with near misses in relation to the behaviors of recovery, reporting, communication, Power Distance and future behavioral changes?

- How do nurses define near misses and recovery behaviors?

- How do nurses react to near misses (i.e., hide, share, document, disseminate)?

- What are practicing nurses' recommendations for clinical practice to reduce the experiences of near misses and strengthen recovery behaviors?

- What are practicing nurses' recommendations for undergraduate nursing curricula in order to improving teaching/learning for safe practices to reduce errors, near misses and recovery behaviors?

\subsection{Purpose}

The purpose of this research study was to investigate how nurses' perceive and experience near misses, error reducing communication, perception of power distance, and recovery behaviors.

\section{LiterATURE REVIEW}

\subsection{Near misses}

Several individual studies have been conducted to define near misses, explore different perceptions of near misses, and examine what causes near misses. A qualitative study was conducted to sharpen the definition of near misses based on empirical data about error handling. ${ }^{[15]}$ These authors wanted to explore whether it was useful to differentiate between incidents that did not reach the patient and incidents that reached the patient but did not cause harm, when defining near misses. ${ }^{[15]}$ Incidents reports and interviews were collected from October 2006 to March 2007 from two Dutch hospitals and a total of 143 cases we reported. The reports and interviews allowed the authors to look at different error recovery methods, which then were used to propose a better definition of near misses. The proposed definition of a near miss was incidents in which successful error recovery prevented the incident from reaching the patient. ${ }^{[15]}$ This study proposed a definition that has several advantages over existing definitions; it's positively stated, it can result in a willingness to report, and it diminishes feelings of shame and fear because no harm was done to the patient.

\subsection{Underreporting}

Negative interpretations of near misses may lead to underreporting such as what happened with the ambulance personnel in the 2010. ${ }^{[22]}$ Data was collected from 11 anonymous Webbased reports, 17 semi-structured interviews, and two focus groups with a total of 23 participants. None of the near misses described by the participants that resulted in potential injury or potential fatality had been previously reported. Participants' stated reasons were that patient outcome were not affected even though an error had occurred, and concerns were expressed about job security. ${ }^{[22]}$ Participants believed that near misses are viewed as negative incidents that can potentially hurt their career of they choose to report.

\subsection{Work factors}

Near misses happen for many reasons depending on the specialty that one works in. In Emergency Medical Technician (EMT) working with pediatric patients, near misses occurred because of problems with procedural skill performance, medication problems/calculations errors, improper equipment size, parental interference, and omission of treatment related to provider's discomfort with the patient's age. ${ }^{[22]}$ In perioperative nursing, near misses are related to team factors, workload factors, task factors, staff factors, patient characteristics, and hospital characteristics. Poor communication 
between team members, distractions, and not following policies were the top three causes of near misses in perioperative nursing. ${ }^{[23]}$ In dialysis nursing, nurses with more knowledge and more experience tend to make fewer mistakes. ${ }^{[24]}$

\subsection{System errors versus human errors}

Errors in health care can be better understood by using Reason's ${ }^{[25]}$ model on human error. Reason describes that with in situations of human error such as medication errors or medication near misses, the error problem can best be divided into two approaches; the person and the system. Both of the approaches have causation that is linked to how best to identify, handle and further prevent errors. With the person approach, quite often one is blamed for lack of skill or attention to detail such as being unmotivated, distracted, careless or forgetful, whereas with the systems approach, all of the environmental and institutional conditions in which the error took place are thought to be tied to a system and workplace, rather than an individual. ${ }^{[25]}$ With either approach, the question remains how best can the error be prevented, or if need be, identified early and rectified. With near misses, nurses might be disinclined to discuss the "almost" error with colleagues, or be hesitant to report the error to administration for follow up, and therefore, neither the person approach nor the systems approach can be assisted or rectified.

\subsection{Emotional impact of errors}

Recovery does not always happen in the face of actual errors. Some have referred to the nurse who committed the error as the "second victim". ${ }^{[26]}$ Here the emotional impact of an error is described to include one's "deeply personal, social, spiritual and professional crisis", ${ }^{[26]}$ especially when the error highly impacts the well-being, or very life, of the patient involved.

The recovery process for a nurse who is emotionally impacted by an error may be smooth, or the road to emotional recover may lead to isolation, doubt in one's ability to perform well in the future and loss of confidence. Emotional distress may continue to the point of remorse, depression and even suicide. ${ }^{[25]}$ Post-error counseling and a focus on job related stress reduction may reduce the incidence of feeling guilty, upset at oneself, and fearful of continued error-prone practice. ${ }^{[27]}$ Coping after an error is essential. Fostering coping may reduce embarrassment and feelings of inadequacy. ${ }^{[28]}$

\subsection{Recovery}

Nursing recovery is defined as a complex process requiring the ability to identify an error as well as to intercede and assist in remedying the situation. This process requires not only the knowledge to recognize an error, also the confidence and Published by Sciedu Press communication skills to address the issue with the appropriate personnel. ${ }^{[29]}$ In nursing, nurses are able to recognize the near miss and correct them. Recovery factors explain why developing incidents did not result in actual accidents. ${ }^{[30]}$ However, the ways nurses recover their near misses varies from person to person and are influenced by many factors such as experience or emotional response to the situation.

An example of an error recovery by Henneman and Gawlinski ${ }^{[29]}$ stated:
"The resident returns the charge nurse's call and gives several telephone orders, including one for administering aspirin. The charge nurse obtains aspirin from the unit stock in the medication room closet, brings it to the bedside, and no- tifies the primary nurse of the new physician orders. The primary nurse checks the orders against the patient record and notes that the pa- tient is allergic to aspirin. She does not adminis- ter the aspirin and notifies the resident when he arrives".

In this example, the primary care nurse successfully identified the error and did not administer the prescribed medication. This was considered a successful error recovery.

Error recovery is defined as the way a person identifies and corrects an error. A study conducted on 20 critical care nurses showed 17 strategies to identify, interrupt, and correct errors. ${ }^{[10]}$ Knowing the patient was identified as an important strategy for identifying an error. Knowing the patient refers to knowing medical and medication histories, obtaining a thorough report from the transfer nurse, and establishing a rapport with the patient's family members. Nurse's ability to interrupt an error has to do with their experiences and confidence. Nurses in this study had a median of 12 years of experience in critical care, and many stated that their inexperience or lack of confidence prohibited them from interrupting an error. These authors also stated that situations in which the nurses could not interrupt a serious error were rare.

Critical to recovery processes is the concept of pattern recognition, defined as the ability of healthcare professionals to recognize cues and decipher patterns in their practice. ${ }^{[31]}$ One nurse stated, "I think it's safer because in recognizing the potential harm to a patient, you want to prevent that as much as possible". Skill and experience are needed to be able to recognize a potential error, and then cognitively processes through the recovery from the potential error. Expertise, or proficiency, is directly linked to one's experience, perceptions, training and preserved knowledge. Expertise 
represents that one has tapped into knowledge and skill, then reveals one's reasoning and judgment. Experts' seasoned experience is linked to social, cognitive and performancerelated factors ${ }^{[32]}$ all of which may aid in pattern recognition, cues, error identification and recovery.

The process of identifying and then correcting near miss errors is still not widely known. Many errors are not being identified, thus leading to near misses, and finally a chance to recovery from that mistake does not always exist. A second analysis by Habraken \& Schaaf ${ }^{[30]}$ emphasized how numerous error recovery opportunities are missed, especially when dealing with medications. Reasons for these missed opportunities are incomplete or unclear protocols that prevented employees from detecting errors, reading drug labels, or heavy workloads in which compliance with safety-related procedures was low. Consequently, required safety checks to prevent near misses or actual errors are not always carried out.

\section{Methods}

\subsection{Sampling procedures}

Active nurses, whose experience ranged from senior students to experienced licensed professionals, who were working clinically in a variety of acute and critical care settings were solicited for participation. Accessible nurses who agreed to participate in the electronic survey between July 15, 2013 and January 6, 2014 were members of national nursing organizations. A sample size of 400 was targeted through random and purposive sampling techniques. Random and purposive sampling techniques were used to collect as many responses as possible from a diverse sample of practicing nurses throughout the country. Those nurses who are actively involved with an accessible professional nursing organization were solicited for participation.

After IRB approval, the research team solicited participation by asking the chapter presidents for access to their electronic mailing lists of members and then made verbal and electronic announcements to members of the professional organizations. Approximately 2,000 potential participants were requested to fill out an electronic survey within the specified time frame with a goal of 400 as a response rate. Purposive sampling also took place. To ensure that both novice and expert nurses participated in the research project, the researchers requested specific advanced practice and seasoned nurses such as all full time and part time faculty at various Bay Area colleges and universities to fill out an electronic survey. Faculty requested to participate held dual roles of clinician and teacher.

Senior nursing students who were in their advanced medical surgical rotation and/or their preceptorship, or last clinical semester, were also asked to participate. Deans or Chairs of the departments of nursing at the Bay Area schools were requested to disseminate the electronic survey web link to all of their senior students. For the purposes of a broader investigation, it was felt that including senior students would add the perspective of novice nurses wo may experience near misses on a regular basis.

\subsection{Electronic sampling procedures}

This research survey used Survey Monkey as a form of electronic data collection. After permission from leaders of professional organizations was secured, a link was provided that allowed for participants to hear about the study aims, answer demographic questions, rating scales, Likert scales, and open-ended questions.

\subsection{Instrument development}

No instrument was identified in previous literature that addressed the complexity of the concepts under investigation. Therefore, an instrument was developed by the research team to specifically address the research questions.

\subsection{Instrument validity and reliability}

Content validity and construct validity were addressed by requesting that three content expert practicing nurses with a doctoral degree and research experience review the instrument. The research team incorporated suggestions made by the reviewers to strengthen the validity of the instrument and ensure the ability of the instrument to be sensitive to the variables under investigation. See Table 2 for a summary of the survey instrument's data collection items. Reliability of the instrument developed for this study was not addressed for this pilot investigation.

\subsection{Procedures}

After institutional review board (IRB) approval from Dominican University of California's Committee on the Protection of Human Rights, data collection began. Leaders from each of the professional organizations were contacted to solicit permission to send a link of the survey to members. Oral presentations took place to the leadership as needed. After securing permission, the link was sent out to members and participation was requested. Two reminders were sent to the members during the course of data collection.

\subsection{Data collection and analyses}

A professional statistician was brought into the research process to provide feedback on the instrument as well as provide guidance on statistical analysis. Descriptive statistical analysis was conducted on the quantitative data. Analysis of qualitative data was done using conventional content analy$\operatorname{sis}^{[33]}$ to analyze the qualitative open-ended data. 
Table 2. Survey instrument

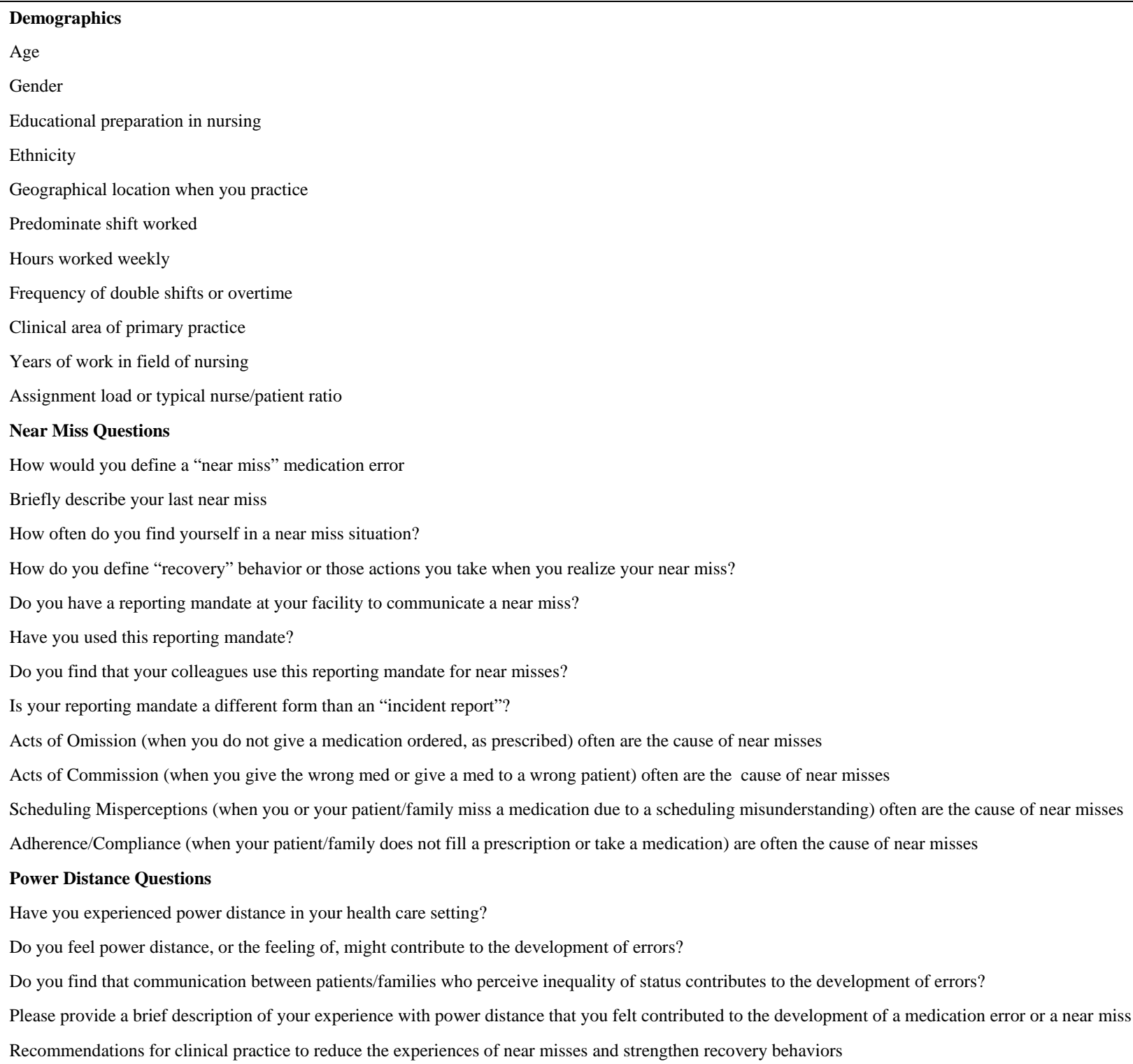

\section{Results}

The response rate of 110 out of 400 targeted for the study represents a $27 \%$ response rate. The nurse participants represented a diverse range of ages, gender, cultural backgrounds, patient care populations, and medical diagnoses. They reported they worked in environments of direct patient care that were prone to medication and medical near misses, actual errors, and had experienced recovery behaviors. See Table 3 for a list of participant demographics. The majority of participants worked in suburban areas (43\%), worked the evening shift (40\%), worked part time (42\%) and identified themselves as medical surgical nurses $(33 \%)$ or undeclared in clinical practice (28\%) (see Tables 4-8).

Analysis of the descriptive data of how near misses are de- fined (see Table 4) demonstrated three general themes of types of near misses experiences: wrong medication to wrong patients; error caught just before reaching patient; and recognition of error, correction made before implementation. The examples shared by the research participants demonstrated that near misses are complex and span across various care behaviors. Examples of near misses were described to be what took place at the moment of care just before the error could have taken place.

Understanding the phenomenon of near misses through the descriptions of study participants can strengthen error reduction strategies by illuminating care behaviors just prior to the error, and acknowledging that emotions are a part of the near miss experience. The definition of near misses was diverse 
and supports previous studies that state no one universal definition of near miss exists.

Table 3. Participant demographics

\begin{tabular}{lll}
\hline Demographics & $\mathbf{N = 1 1 0}$ & $\mathbf{( \% )}$ \\
\hline Age & $22-65$ & \\
$\quad$ Range & 28 & \\
Mean & & \\
Gender & 11 & $10 \%$ \\
$\quad$ Male & 98 & $89 \%$ \\
Female & 1 & $1 \%$ \\
Rather not disclosed & & \\
Education & 2 & $2 \%$ \\
$\quad$ AA & 96 & $87 \%$ \\
BSN & 12 & $11 \%$ \\
MSN & & \\
Ethnicity & 10 & $9 \%$ \\
Latino/Hispanic & 52 & $47 \%$ \\
Caucasian & 20 & $18 \%$ \\
Filipino & 12 & $11 \%$ \\
Asian & 1 & $1 \%$ \\
African American & 1 & $1 \%$ \\
Multi-racial & 14 & $13 \%$ \\
Undeclared & & \\
\hline
\end{tabular}

Thematic analysis on last near miss examples (see Table 5) disclosed six themes related to actual examples of near misses: patient identified the near miss; medication was already administered by another nurse; a systems error occurred with the computerized dispending unit; the five rights were breeched; timing of administration was incorrect; and the wrong medication was dispensed directly from the phar- macy. The data demonstrate that adhering to the 5 rights reduces the risk of near misses.

Table 4. Thematic results: How do you define near misses?

Identified Themes:
Wrong medication to wrong patient
Error caught before reaching patient
Recognition of error, correction made before implementation
Thematic Examples:
"When a nurse realizes that they are about to administer a
medication that is not correct - one of the 5 rights is wrong"
"The error is about to be initiated, but recognition of errors is
noted and the correction is made"
"An error that was prevented; a near miss"
"Mortified, panicked, found out in the room"
"It's my own impatience that causes my near misses"
"Constant interruption while performing a med pass"
"Not good"

Three themes (see Table 6) emerged concerning miscommunication and near misses: between healthcare team members; between shifts; and pharmacy verifying and approving wrong med/wrong dose. Power Distance, overall, was only minimally found to be of concern to the participants in relation to near misses.

Table 7 shows definitions of recovery and represents how nurses cope with near misses. Themes included emotional reactions (visceral reactions), the need to talk about the near miss, and how it takes time to recover. Table 8 demonstrates the nurses' experience with power distance including the themes of not speaking up, do not question authority, and fear of clarifying misunderstandings.

Table 5. Thematic results: Briefly describe your last near miss?

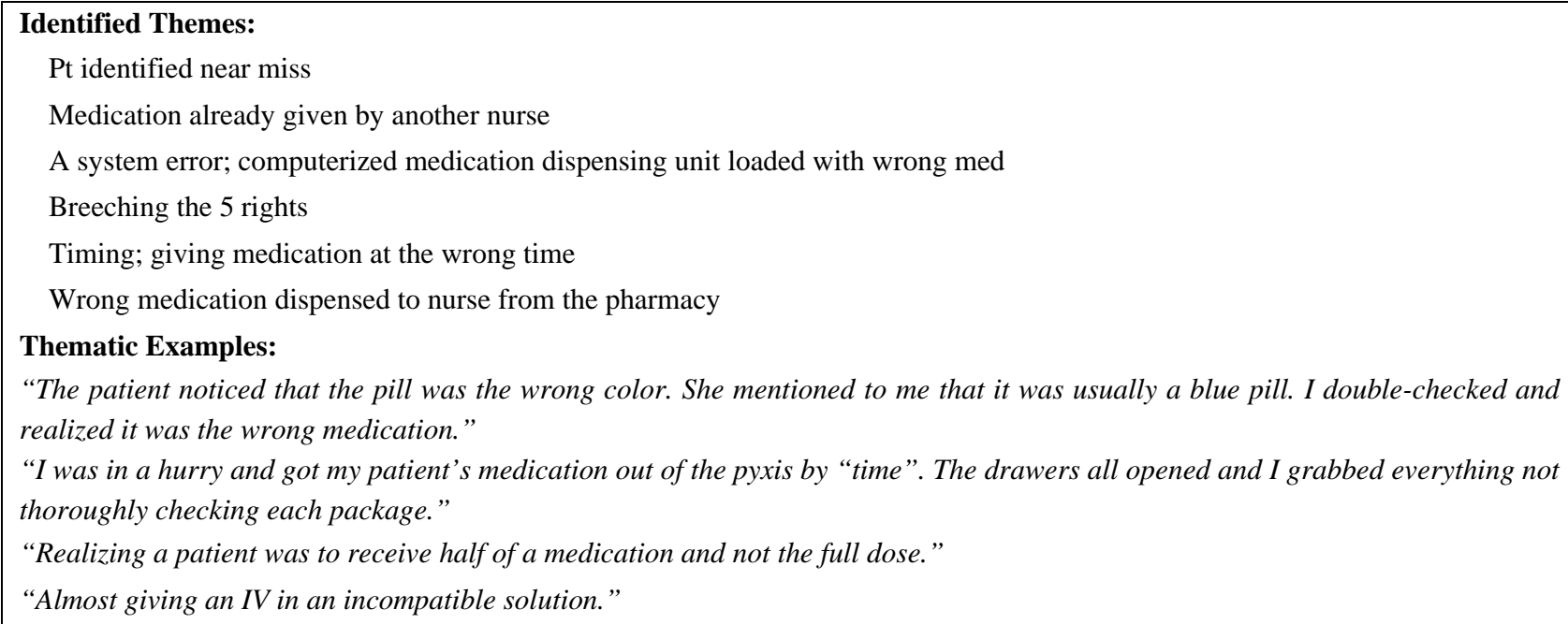


Table 6. Thematic results: Explain the miscommunication that happened

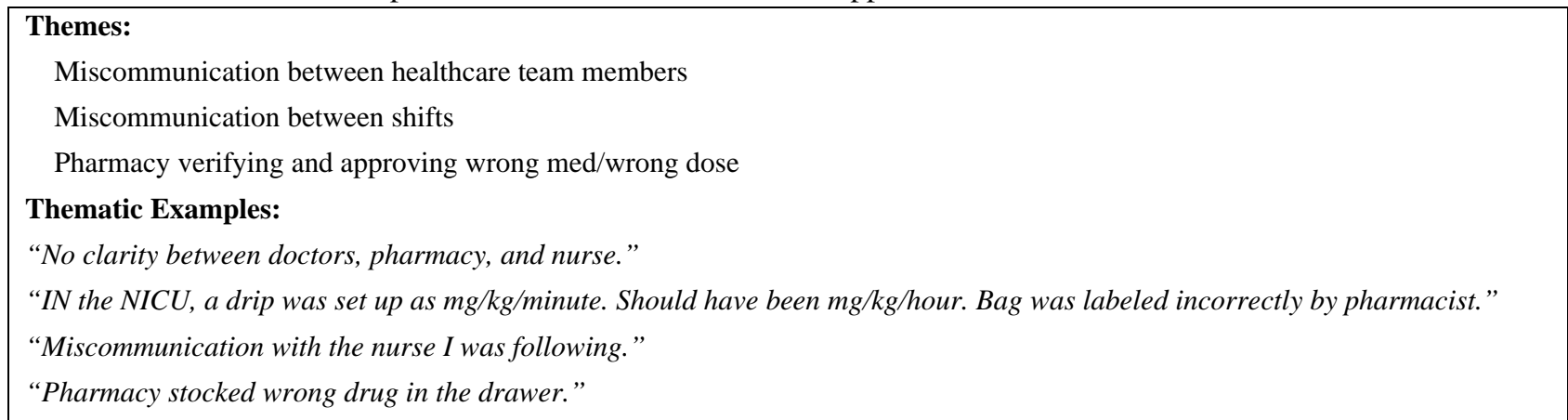

Table 7. Thematic results: Definition of recovery

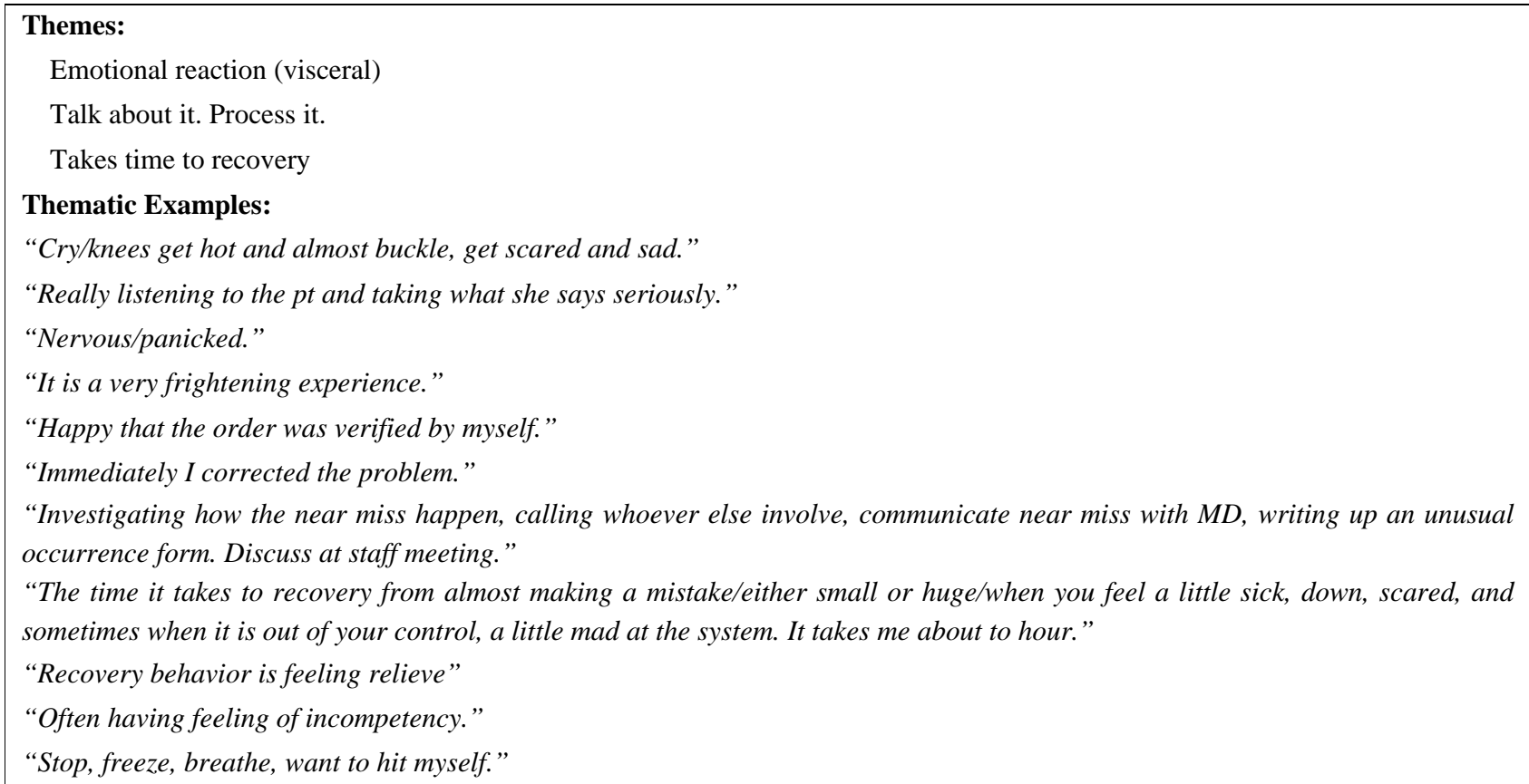

\section{Conclusions}

The investigation has confirmed that near misses are a frequently encountered clinical phenomena that warrants attention. This study illuminated that the types of near misses experienced by the participants closely match those that are described in other previous studies including breeches of the five rights, inaccurate timing of administration, and systems errors. Miscommunication between health care providers, between shifts during handoffs (report) and verification processes continue to contribute to both near misses and errors. The concept of Power Distance, ${ }^{[34]}$ although described by over half of the participants as real and concerning, was not found to be of as much concern with near misses as actual medication errors. When Power Distance was involved, the reluctance of the patients and their families to clarify or be Published by Sciedu Press honest about not understanding what was being discussed contributed to medication errors after discharge.

\section{Recommendations for clinical practice and education}

Participants provided diversity in suggestions and recommendations for both clinical practice and curriculum that would help nurses understand, identify and develop strategies for reducing actual errors, near misses and errors related to communication and power distance. Recommendations for curriculum for undergraduate nursing programs included the addition of more simulations focused on safety and error reduction as well as simulations that will allow students to "Come to a crossroad where they must make decisions" and experience "Error-rich or error-prone simulations where the students have at least a 3 patient assignment." Participants suggested; "Creating a skills lab that have live people 
who are loud, who are talking, interacting, and breathing." Other suggestions for curriculum included learning to listen carefully, role play clinical errors and experience the stress and turbulence of complex adaptive systems. One specific example was to: "Have someone refuse medical treatment (transfusion therapy) such as a member of the Jehovah Witness church."

Table 8. Thematic results: Experiences of power distance and near misses or errors

Themes:
Not speaking up
Do not questions authority
Fear of clarifying misunderstandings
Thematic Examples:
“Communication, or lack of, misinformation, patients' reluctance to ask for clarification and providers reluctance to check for
understanding... all play a role in near misses and errors."
"Oh yes... in think often in the NICU world, parents feel like they know little to nothing, so they don't speak up for their baby when
they think there may be an error in progress..."
"Yes, I see this a lot. Many times it is when the families do not question the authority of the health care provider or they do not speak
up and ask question or say something they should. They just shake their head yes and agree with everything..."
"Power distance is something like when prescriptions are made without the full buy in of the patient... they are going to say yes and
sure, and then not ask questions and not fill prescriptions...”
"My pateints are afraid to ask questions... they don't understand all aspects of medications and treatments and they won't ask
questions... they are reluctant to ask questions...
"Oh yes... in my area... many of my patients come from low income status... the family member understand that the nurses give the
treatments in the hospital however when the patents bring their infants home the medications are spaced out every other day to save
money, time an ease... this is not brought back to the doctor....

Recommendations for the practice of nursing included the topics of time management, improved reporting systems, adherence to the five rights, avoidance of being in a hurry, double checking and being kind and supportive to one another, providing continuing education classes on safety and error reduction, and never skipping on the two nurse check or two nurse independent verification of calculations.

The research team recommends that nurses who experience near misses should be given support to process their emotional reactions and their need for time for recovery. Nurses should be offered counseling and opportunities to process their experiences while continuing to provide patient care. Nurses should be allowed to investigate the individual and system errors that led to the near miss. Further research is needed to investigate best practices for supporting nurses through recovery from near misses. Also, further research is needed to investigate best practices on investigating the development of near misses.

\section{Discussion}

Although many new advanced technological improvements are now available to reduce the incidence of errors, human factors continue to influence the continuation of near misses and medication errors. It is imperative that nurses are aware of factors that contribute to errors and continue to seek innovative ways to compliment new technology to reduce near misses. Power distance, or the perception that inequality exists, has an impact on both near misses and actual errors when communication is a factor, but was only minimally described to be an influencing factor. Further research is needed beyond this pilot study to investigate individual, systems, and communication contributions to near misses and recovery behaviors.

\section{Limitations}

Several limitation were identified in this study. The use of purposive sampling was done to secure data from nurses that represent various care areas and length of practice. This sampling technique weakens generalizing the results beyond the sample. The goal of 400 participants was reduced to only 110 actual respondents. This smaller sample size also reduces the generalizability of the results. Replication of the study with a larger sample size and the separation of data between senior nursing students, early professionals in practice and those very experienced in practice may provide deeper understanding of how communication, clarification and Power Distance influence the development of near misses. 


\section{REFERENCES}

[1] Institute of Medicine. Crossing the quality Chasm: A new health system for the 21st century. 2006.

[2] Star K, Nordin K, Pöder U, et al. Challenges of safe medication practice in paediatric care - A nursing perspective. Acta Paediatrica. 2013; 102(5): 532-538. http://dx.doi.org/10.1111/apa. 12212

[3] Wang Hf, Jin JF, Feng XQ, et al. Quality Improvements in Decreasing Medication Administration Errors Made By Nursing Staff in an Academic Medical Center Hospital: A trend analysis during the journey to Joint Commission International Accreditation and in the post-accreditation era. Therapeutics and Clinical Risk Management. 2015; 11: 393-406.

[4] Kohn LT, Corrigan JM, Donaldson MS. To err is human: Building a safer health system. Washington, DC: National Academy Press Institute of Medicine; 2000.

[5] Stelfox H, Palmisani S, Scurlock C, et al. The "To Err is Human" Report and the Patient Safety Literature. Quality and Safety in Health Care. 2006; 15(3): 174-178. PMID:16751466. http: //dx.doi.org/10.1136/qshc. 2006.017947

[6] Härkänen M, Turunen H, Saano S, et al. Medication errors: What hospital reports reveal about staff views. Nursing Management - UK. 2013; 19(10): 32-37. http://dx.doi.org/10.7748/nm2013.03 .19.10.32.e1010

[7] Hofstede G. Culture's consequences: comparing values, behaviors, institutions, and organizations across nations. (2nd ed.). Thousand Oaks, CA: Sage Publications; 2001.

[8] Hofstede G, Hofstede GJ, Minkov M. Cultures and organizations. Software of the mind. (3rd ed.). New York, NY: McGraw Hill; 2005.

[9] Richardson RM, Smith SW. The influence of high/low-context culture and distance on choice of communication media: Students' media choice to communicate with Professors in Japan and America. International Journal of Intercultural Relations. 2007; 31: 479-501. http://dx.doi.org/10.1016/j.ijintrel.2007.01.002

[10] Henneman EA, Gawlinski A, Blank FS, et al. Strategies used by critical care nurses to identify, interrupt, and correct medical errors. American Journal of Critical Care. 2010; 19(6): 500-509. http://dx.doi.org/10.4037/ajcc2010167

[11] Moran RT, Harris PR, Moran S. Managing cultural differences: Global leadership strategies for the 21st century. (8th ed). Burlington, MA: M Elsevier Butterworth Heinemann; 2010.

[12] Rudd JE, Lawson DR. Communication in global business negotiations. A geocentric approach (1st ed.). Thousand Oaks, CA: Sage; 2007.

[13] Thomas DC. Cross-Cultural management essential concepts. (2nd ed.). Thousand Oaks, CA: Sage; 2008.

[14] Knudsen P, Herborg H, Mortensen AR, et al. Preventing Medication Errors in Community Pharmacy: Frequency and seriousness of medication errors. Quality and Safety in Health Care. 2007; 16(4): 291-296. http://dx.doi.org/10.1136/qshc.2006.018770

[15] Kessels-Habraken M, Van der Schaaf T, De Jonge J, et al. Defining near misses: Towards a sharpened definition based on empirical data about error handling processes. Social Science \& Medicine. 2010; 70(9): 1301-1308. http://dx.doi.org/10.1016/j.socscimed .2010 .01 .006

[16] Barnard D, Dumkee M, Bains B, et al. Implementing a good catch program in an integrated health system. Healthcare Quarterly. 2006; 9(Sp): 22-27. http://dx.doi.org/10.12927/hcq.2006.183 73

[17] Kaplan HS, Rabin Fastman B. Organization of event reporting data for sense making and system improvement. Quality and Safety in Health Care. 2003; 12(Suppl.2): ii68-ii72. http://dx . doi.org/1 $0.1136 /$ qhc.12.suppl_2.ii68
[18] Barach P, Small SD. Reporting and preventing medical mishaps: lessons from non-medical near miss reporting systems. British Medical Journal. 2000; 320: 759-763. PMID:10720361. http://dx.doi .org/10.1136/bmj.320.7237.759

[19] Barach P, Small SD, Kaplan H. Designing a confidential safety reporting system: in depth review of thirty major medical incident reporting systems, and near-miss safety reporting systems in the nuclear, aviation, and petro-chemical industries. Anesthesiology. 1999; 91: A1209.

[20] Gurwitz J, Field T, Avorn J, et al. Incidence and Preventability of Adverse Drug Events in Nursing Homes. The American Journal of Medicine. 2000; 109(2); 87-94. http://dx.doi.org/10.1016/S 0002-9343(00) 00451-4

[21] Aspden P, Corrigan J, Wolcott J, et al. Patient safety: Achieving a new standard for care. Washington, DC: National Academies Press; 2004.

[22] Cushman JT, Fairbanks RJ, O'Gara K, et al. Ambulance personnel perceptions of near misses and adverse events in pediatric patients. Prehospital Emergency Care. 2010; 14(4): 477-484. http: //dx.doi.org/10.3109/10903127.2010.497901

[23] Cohoon B. Causes of near misses: Perceptions of perioperative nurses AORN Journal. 2011; 93(5): 551-565. http://dx.doi.org/10 1016/j . aorn. 2010.02.017

[24] Wilkinson WE, Cauble LA, Patel VL. Error detection and recovery in dialysis nursing. Journal of Patient Safety. 2011; 7(4): 213-223. http://dx.doi.org/10.1097/PTS . Ob013e3182388d20

[25] Reason J. Human Error: Models and management. British Journal of Medicine. 2000; 320(7237): 768-770. http://dx .doi .org/10. $1136 / \mathrm{bmj}$. 320.7237.768

[26] Institute for Safe Medication Practices. Too Many Abandon the "Second Victims" of Medical Errors. 2011.

[27] Waterman A, Garbutt J, Hazel E, et al. The Emotional Impact of Medical Errors on Practicing Physicians in the United States and Canada. The Joint Commission on Accreditation of Healthcare Organizations 2007; 33(8): 467-476.

[28] White A, Gallagher TH. After the Apology- Coping and Recovery after Errors. AMA Journal of Ethics. Virtual Mentor. 2011; 13(9): 593600. PMID:23137470. http://dx.doi.org/10.1001/virtual mentor.2011.13.9.ccas1-1109

[29] Henneman EA, Gawlinski A. A "near-miss" model for describing the nurse's role in the recovery of medical errors. Journal of Professional Nursing. 2004; 20(3): 196-201. PMID:15211429. http://dx.doi.org/10.1016/j.profnurs.2004.04.006

[30] Habraken M, Schaaf T. If only....: Failed, missed and absent error recovery opportunities in medication errors. Quality \& Safety in Health Care. 2010; 19(1): 37-41. http://dx.doi.org/10.1136/qshc. 2007.026187

[31] Jeffs L, MacMillan K, Maione M. Leveraging safer nursing care by conceptualizing near misses as recovery processes. Journal of Nursing Care Quality. 2009; 24(2): 166-171. http://dx.doi.org /10.1097/01.NCQ.0000347455.21171.49

[32] Hoffman R, Shadbolt N, Burton AM, et al. Eliciting Knowledge from Experts: a methodological analysis. Organizational Behavior and Human Decision Processes. 1995; 62(2): 129-158. http: //dx.doi.org/10.1006/obhd.1995.1039

[33] Hsieh HF, Shannon S. Three Approaches to Qualitative Content Analysis. Qualitative Health Research. 2005; 15(9): 1277-1288. PMID:16204405. http://dx.doi.org/10.1177/10497323052 76687

[34] Hofstede G. Culture's consequences: International differences in work-related values. Beverly Hills, CA: Sage; 1980. 